\title{
SOMO: Self-Organized Metadata Overlay for Resource Management in P2P DHT
}

\author{
Zheng Zhang \\ Microsoft Research China \\ zzhang@microsoft.com
}

\begin{abstract}
In this paper, we first describe the concept of data overlay, which is a mechanism to implement arbitrary data structure on top of any structured P2P DHT. With this abstraction, we developed a highly scalable, efficient and robust infrastructure, called SOMO, to perform resource management for P2P DHT. It does so by gathering and disseminating system metadata in $O(\log N)$ time with a selforganizing and self-healing data overlay. Our preliminary results of using SOMO to balance routing traffic with node capacities in a prefix-based overlay have demonstrated the utility of data overlay as well as the potential of SOMO.
\end{abstract}

\section{Introduction}

For a large $\mathrm{P} 2 \mathrm{P}$ overlay to adapt and evolve, there must be a parallel infrastructure to monitor the health of the system (e.g. "top"-like utility in UNIX). The responsibility of such infrastructure is to gather from and distribute to entities comprising the system whatever system metadata of concern, and possibly serve as the channel to communicate various scheduling instructions. The challenge here is that this infrastructure must be embedded in the hosting DHT but is otherwise agonistic to its specific protocols and performance; it must grow along with the hosting DHT system; it must also be fault resilient and, finally, the information gathered and/or disseminated should be as accurate as possible.

In this paper, we describe the Self-Organized Metadata Overlay, or SOMO in short, which accomplishes the above goal. By using hierarchy as well as soft-state, SOMO is selforganizing and self-healing, and can gather and disseminate information in $\mathrm{O}(\log N)$ time. SOMO is simple and flexible, and is agnostic to both the hosting P2P DHT and the data being gathered and disseminated. The later attribute allows it to be programmable, invoking appropriate actions such as merge-sort and aggregation as data flows through.

Through the development of SOMO, we have discovered that there is a consistent and simple mechanism to implement arbitrary data structure on top of a P2P DHT. We refer to a data structure that is distributed onto a DHT a data overlay. Data overlay is discussed in Section-2. Following that, we describe the construction and operations of SOMO in Section3 , and also its application in Section-4. A case study of using SOMO to balance routing traffic to node capacity in a prefixbased overlay is offered in Section-5, along with preliminary

* Work done as intern in MSR-Asia.
Shu-Ming Shi* and Jing Zhu*

CS Dept. Tsinghua University

\{ssm99, zhujing00\}@mails.tsinghua.edu.cn

results. Related works are discussed in Section-6, and we conclude in Section-7.

\section{Data Overlay: Implement Arbitrary Data Structures on top of P2P DHT}

We observe that hash-table is only one of the fundamental data structures. Sorted list, binary trees and queues etc. all have their significant utilities. One way would be to investigate how to make each of them self-organized (i.e., P2P sorted list). Another is to build on top of a hash table that already has the self-organizing property (i.e. P2P DHT). This second approach, which we call data overlay, is what we take in this paper.

Any object of a data structure can be considered as a document. Therefore, as long as it has a key, that object can be deposited into and retrieved from a P2P DHT. Objects relate to each other via pointers, so to traverse to the object $b$ pointed by $a$.foo, a.foo must now store $b$ 's key instead. More formally, the following two are the necessary and sufficient conditions:

- Each object must have a key, obtained at its birth

- If an attribute of an object, a.foo, is a pointer, it is expanded into a structure of two fields: a.foo.key and a.foo.host. The first substitutes the hard-wired address of a conventional pointer; and the second field is a soft state containing the last known hosting DHT node of the object $a$.foo points to and serves as a routing shortcut.

It is possible to control the generation of object's key to explore data locality in a DHT. For instance, if the keys of $a$ and $b$ are close enough, it's likely that they will be hosted on the same machine in DHT.

We call a data structure distributed in a hosting DHT a data overlay. It differs from traditional sense of overlay in that traversing (or routing) from one entity to another uses the free service of the underlying P2P DHT.

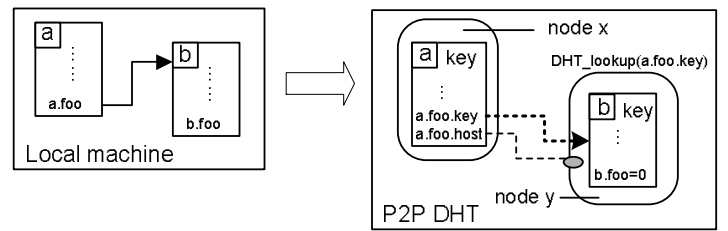

Figure 1: implement arbitrary data structure in DHT.

Figure 1 contrasts a data structure in local machine versus that on a P2P DHT. Important primitives that manipulate a pointer in a data structure, including setref, deref (dereferencing) and 
delete, are outlined in Figure 2. Here, we assume that both DHT_lookup and DHT_insert will, as a side effect, always return the node in DHT that currently hosts the target object. DHT_direct bypasses normal DHT_lookup routing and directly seeks to the node that hosting an object given its key.

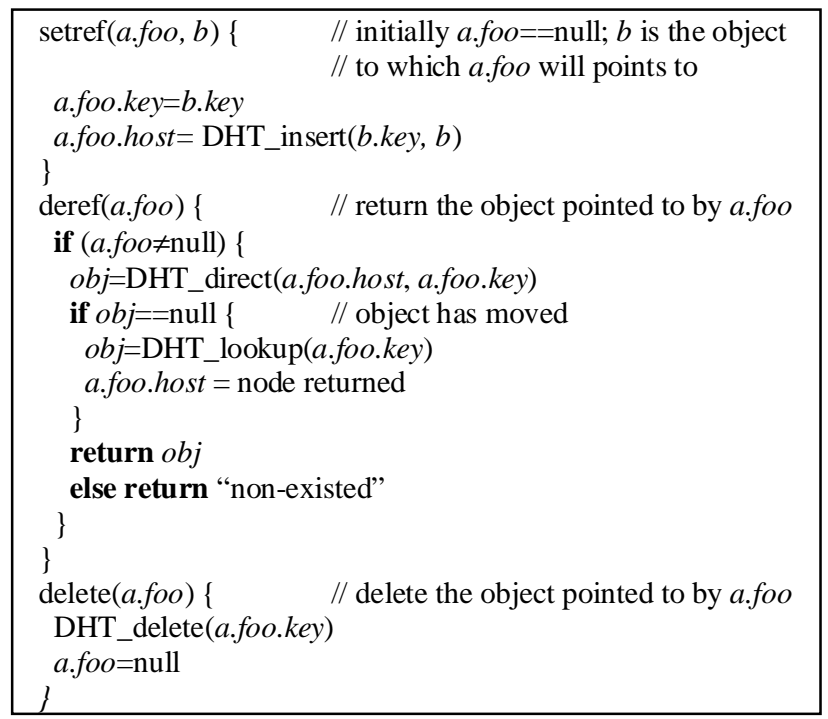

Figure 2: pointer manipulate primitives in data-overlay

The interesting aspect is that it is now possible to host any arbitrary data structure on a P2P DHT, and in a transparent way. What need to be modified are the library routine that creates an object to insert a key as its member, and the few primitives that manipulate pointers as outlined. Therefore, legacy applications can be ported to run on top of a P2P DHT, giving them the illusion of an infinite storage space (here storage can broadly include memory heaps of machines comprising the DHT). The host routing shortcut makes the performance independent of the underlying DHT when the overall system dynamism is small.

A data overlay on top of a bare-bone P2P DHT with no internal reliability support can be used to implement distributed data structure that is soft-state in nature (i.e,, data is periodically refreshed and consumed thereafter without ill side-effect). If advanced fault-tolerant techniques are employed, then data overlay can spawn even more interesting research threads. For instance, it maybe possible to turn DHT into a parallel computing utility by building a globally accessible and fully-associative memory heap that as a repository of shared-variables [14].

\section{Self-Organized Metadata Overlay}

We now describe the data overlay SOMO (Self-Organized Metadata Overlay), an information gathering and disseminating infrastructure on top of any P2P DHT. Such an infrastructure must satisfy a few key properties: selforganizing at the same scale as the hosting DHT, fully distributed and self-healing, and be as accurate as possible of the metadata gathered.

Such metadata overlay can take a number of topologies. Given that one of the most important functionalities is aggregation, our implemented SOMO is a tree of $k$ degree whose leaves are planted in each DHT node. Information is gathered from the bottom and propagates towards the root, and disseminated by trickling downwards. Thus, one can think of SOMO as doing converge cast from the leaves to the root, and then multicast back down to the leaves again. Both the gathering and dissemination phases are $\mathrm{O}\left(\log _{k} N\right)$ bounded, where $N$ is total number of entities. Each operation in SOMO involves no more than $k+1$ interactions, making it fully distributed. We deal with robustness using the principle of soft-state, so that data can be regenerated in $\mathrm{O}\left(\log _{k} N\right)$ time. The SOMO tree selforganizes and self-heals in the same time bound. We now explain the details of SOMO.

\subsection{Building SOMO}

Since SOMO is a tree, we call its node the SOMO node. To avoid confusion, we denote the DHT nodes as simply the DHT node. A DHT node that hosts a SOMO node $s$, is referred to as DHT_host $(s)$.

struct SOMO_node \{
string key
struct SOMO_node $*$ child $[1 . . k]$
DHT_zone_type $Z$
SOMO_op op
Report_type report
\}

\section{Figure 3: SOMO node data structure}

The basic structure of the type SOMO_node is described in Figure 3. The member $Z$ indicates the region of which this node's report member covers. Here, the region is simply a portion of the total logical space of the DHT. The root SOMO node covers the entire logical space. The key is produced by a deterministic function of a SOMO node's region $Z$. Examples of such functions include the center of the region, or a hash of the region coordinates (see Figure 4). Therefore, a SOMO node $s$ will be hosted by a DHT node that covers s.key (e.g. the center of $s . Z$ ). This allows a SOMO node to be retrieved deterministically as long as we know its region and is particularly useful when we want to query system status in a given key-space range. A SOMO node's responsible region is further divided by a factor of $k$, each taken by one of its $k$ children, which are pointers in the SOMO data structure. A SOMO node $s$ 's $i$-th child will cover the $i$-th fraction of region $s . Z$. This recursion continues until termination condition is met (discussed shortly), and since a DHT node will own a piece of the logical space, it is therefore guaranteed a leaf SOMO node will be planed in it.

Initially, when the system contains only one DHT node, there is only the SOMO root. As the DHT system grows, SOMO builds its hierarchy along. This is done by letting each SOMO node periodically execute the routine SOMO_grow, shown in Figure 4.

We test first if the SOMO node's responsible zone is smaller or equal to that of the hosting DHT node, if the test comes out to be true, then this SOMO node is already a leaf planted in the right DHT node and there is no point to grow any more children. Otherwise, we attempt to grow. Note that we initialize a SOMO node object and its appropriate fields, and 
then call the setref primitive (See Figure-2) to install the pointer; this last step is where DHT operation is involved.

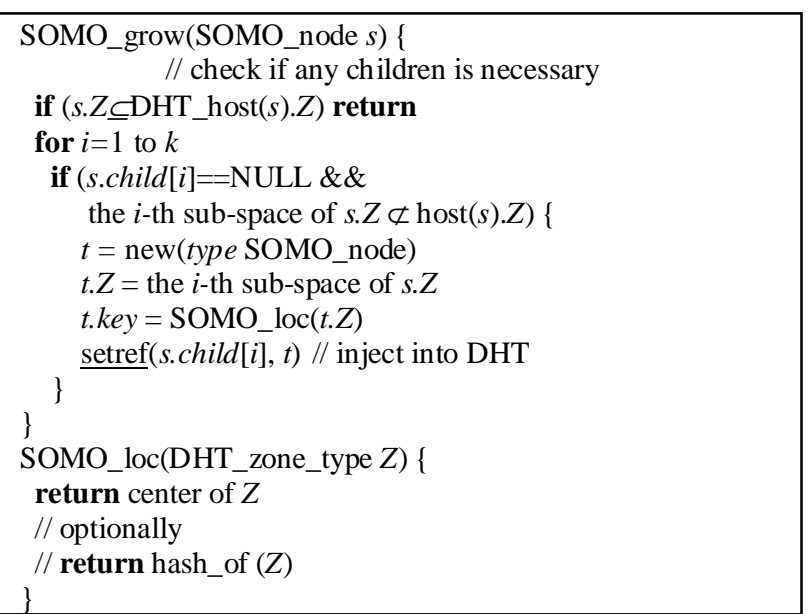

Figure 4: SOMO_grow procedure and the SOMO_loc procedure which deterministically calculates a SOMO node's key given the region it covers.

As this procedure is executed by all SOMO nodes, the SOMO tree will grow as the hosting DHT grows, and the SOMO tree is taller in logical space regions where DHT nodes are denser. This is illustrated in Figure-5.

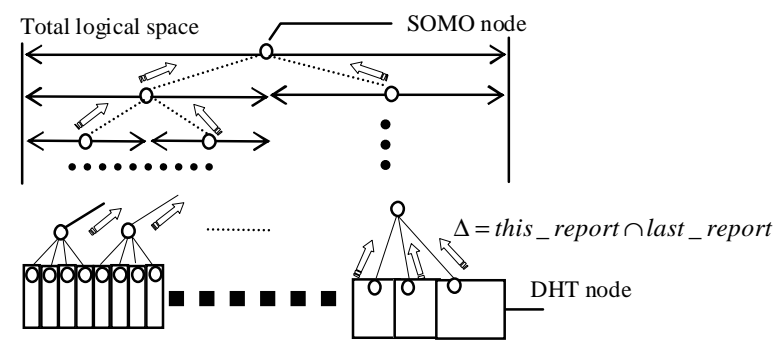

Figure 5: SOMO tree on top of P2P DHT

The procedure is done in a top down fashion, and is executed periodically. A bottom-up version can be similarly derived. When the system shrinks, SOMO tree will prune itself accordingly by deleting redundant children. For an $N$-node system where nodes populate the total logical space evenly, there will be $2 N$ SOMO-nodes when the SOMO fan-out $k$ is 2 .

The crash of a DHT node will take away the SOMO nodes it is hosting. However, the crashing node's zone will be taken over by another DHT node after repair. Consequently, the periodical checking of all children SOMO nodes ensures that the tree can be completely reconstructed in $\mathrm{O}\left(\log _{k} N\right)$ time. Because the SOMO root is always hosted by the DHT node that owns one deterministic point of the total space, that node ensures the existence of the SOMO root and invokes the SOMO_grow routine on the SOMO root.

\subsection{Gathering and disseminate information with SOMO}

To gather system metadata, for instance loads and capacities, a SOMO node periodically requests report from its children. The leaf SOMO nodes simply get the required info from their hosting DHT nodes. As a side-effect, it will also re-start a child SOMO node if it has disappeared because the hosting DHT node's crash. Figure 5 illustrates the procedure.

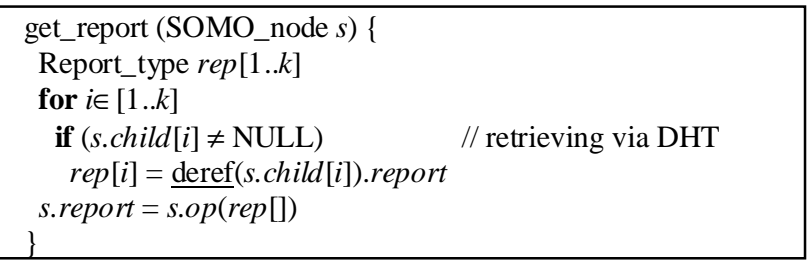

\section{Figure 5: SOMO gathering procedure}

The routine is periodically executed at an interval of $T$. Thus, information is gathered from the SOMO leaves and flows to its root with a maximum delay of $\log _{k} N \cdot T$. This bound is derived when flows between hierarchies are completely unsynchronized. If upper SOMO nodes' call immediately triggers the similar actions of their children, then the latency can be reduced to $T+t_{\text {hop }} \cdot \log _{k} N$, where $t_{\text {hop }}$ is average latency of a trip in the hosting DHT. The unsynchronized flow has latency bound of $\log _{k} N \cdot T$, whereas the synchronized version will be bounded by $T$ in practice (e.g., 5 minutes). Note that $\mathrm{O}\left(t_{\text {hop }} \cdot \log _{k} N\right)$ is the absolute lower bound. For $2 \mathrm{M}$ nodes and with $k=8$ and a typical latency of $200 \mathrm{~ms}$ per DHT hop, the SOMO root will have a global view with a lag of 1.6s.

Dissemination using SOMO is essentially the reverse: data trickles down through the SOMO hierarchy towards the leaves. Performance is therefore similar to gathering. By some modification, dissemination can piggyback on the return message in the gathering phase. The other alternative is to query the SOMO tree. Since SOMO is hierarchical, it is easy to form complex range queries to discover information relevant to a given logical space region. For example, if $k$ is 2 and we wish to get status report of the first $1 / 4$ of the space, we need only to obtain report from the left child of the $2^{\text {nd }}$ level SOMO tree. An even more interesting alternative will be to register queries at SOMO nodes, which essentially transforms SOMO into a pub/sub infrastructure.

Operations in either gathering or disseminating phases involve one interaction with the parent, and then with $k$ children. Thus, the overhead in a SOMO operation is a constant. The entities involved are the DHT nodes that host the SOMO tree. SOMO nodes are scattered among DHT nodes and therefore SOMO processing is distributed and scales with the system.

It seems that towards the SOMO root the hosting DHT nodes need to have increasingly higher bandwidth and stability. As discussed earlier, stability is not a concern because the whole SOMO hierarchy can be recovered in $\mathrm{O}\left(\log _{k} N\right)$ time. As for bandwidth, most of the time one needs only to submit delta between reports (Figure 5). Compression will further bring down message size. Finally, it is always possible to locate an appropriate node through SOMO. This node can swap with the one who is hosting the SOMO root currently. That is to say, SOMO can be self-optimizing as well.

\subsection{Discussion}

The power of SOMO lies in its simplicity and flexibility: it specifies neither the type of information it should gather and/or disseminate, nor the operation invoked to process them. 
That is to say, SOMO operations are programmable and active. For this reason, in the pseudo-code we have used $o p$ as a generic notation for operation used. Using the abstraction of data overlay (especially the host routing shortcut), its performance is also insensitive to the hosting DHT.

We have described SOMO in a collaborative environment to start with. If there are malicious nodes, then the trustworthiness of SOMO itself is under doubt. We offer some of our preliminary thoughts here:

- Denial-of-service attacks can be mounted by relentlessly requesting SOMO root. We believe an efficient way to defend against this is by propagating copies throughout the network, thereby stopping the attacks on the system edge. This borrows the idea from Freenet[2].

- SOMO reports can be compromised in multiple ways on the paths of aggregation and dissemination. To guard against this, reports must be signed and redundant SOMO reports need to be generated through multiple SOMO internal nodes, and use voting for consensus and intruder detection.

- Finally, the most difficult attack to solve is that individual node can simply cheat about its own status. Feedback processes among peers external to SOMO should be used to establish trustworthiness of each node.

\section{Application of SOMO}

As a scalable, fault-tolerant metadata gathering and dissemination infrastructure, the utilities of SOMO are many. In a large scale system, the need to monitor the health of the system itself can not be understated. We have implemented a SOMO-based global performance monitor with which we monitor the servers in our lab on a daily basis. This tool employs SOMO built over a P2P DHT and gathers data from various performance counter on each machine and presents a unified UI interface to clients. We tested the SOMO stability by unplugging cables of servers being monitored, and each time the global view is regenerated after a short jitter. Using the data overlay abstraction, the SOMO layer is implemented much like any local procedures, with only a few hundred lines of code.

More advanced usages are chiefly decided by algorithms that built upon the metadata that gathered. It is possible to build a SOMO on top of a basic, mesh-based P2P DHT, and then build a $\mathrm{O}(\log N)$ soft-state prefix-based overlay by installing long-range entries because SOMO provides the knowledge of what nodes exist in what portion of the total logic space. Even more useful is the fact that SOMO can create an image of a single resource pool comprised of nodes forming the DHT.

Another instance would be to find powerful nodes, commonly known as supernodes. To do this, we will make a SOMO tree where the report type is sorted list, and the $o p$ is merge-sort. Thus, SOMO can mine out multiple classes of supernodes, as reports available at various internal SOMO nodes, with the SOMO root having the complete list. These supernodes can thus act as indexing [6] or routing hobs [17]. There are also proposals where routing performance is the best but storage uniformity is sacrificed [8], SOMO can discover the density map of node capacities. Such information can guide document placement, or migrate nodes from dense regions to weak ones. In this way, uniformity will improve over time. We have also mentioned the possibility of turning SOMO into a pub/sub infrastructure.

\section{Case Study: Balancing Routing Power with Routing Traffic in Prefix-Based Overlay}

Prefix-based overlay includes Tapestry[16], Pastry[9], Chord[11], Kademlia[5] and eCAN[13] (CAN[7] with simple extension). Though some aspects of these proposals differ, they share a few key attributes: 1) the total logical (or key) space is recursively divided and 2) routing greedily seeks out the biggest span into a sub-space and then zoom in towards target quickly. Routing table of prefix-based overlay is an array, recording spaces of exponentially decreasing size and one or several nodes that serve as this node's gateway, or "router" into these spaces. The flexibility of the prefix-based overlays is that, any node in the target sub-space can be a router. This gives rise to many optimization opportunities. Pastry and eCAN explore the possibilities of using the geographically closest node as router candidates to improve routing performance. In this paper, we report our investigation on another complementary axis: choosing the more powerful nodes to serve as routing entrances for larger sub-spaces where traffics are exponentially more than sub-spaces further enclosed. The ultimate solution (and challenge) of selecting these "routers" is to consider all the following three factors: geographic vicinity, routing capacity and load distribution. This remains to be one of our future works.

Our goal is to promote the most capable nodes to handle traffics into larger space, relieving weaker nodes off these responsibilities. Intuitively, the most powerful nodes will take the bulk of the loads in the largest enclosing space, and the weaker ones will serve no more than those designated to its immediate neighbor. Due to space limitation, we refer readers to [15] for the full protocol. Our basic idea is to classify routing loads that a node takes according to the sub-space in which the routing is designated and divide a node's routing capacity accordingly. The end-goal is that each sub-space's load/capacity ratio approaches that of the whole system.

Our optimization consists of four algorithms:

- Statistic collection algorithm. Aggregate loads and capacity statistics in a bottom-up sweep through SOMO. The goal is to have a "view" of the demographic distribution of both loads and capacities. At this point, the load/capacity ratios of the whole system as well as all enclosing spaces are available.

- Load balance algorithm. Top-down sweep to determine the amount of routing capacities to be dedicated in each space, so that its load/capacity ratio approaches to that of the whole system where possible.

- Capacity selection algorithm. Select the right portion of capacities, as recommended by the previous step, from candidate nodes. Also bottom-up sweep. At the end of this 
algorithm, we have selected the right capacity divide responsible for traffic loads of different space.

- Entries dissemination algorithm. Notify other nodes to use these new "routers" so that load distribution can take effect.

The core of our algorithm is in the 2 nd and the 3rd step. It can be simplified if not for a subtle but important issue. The load and capacity distribution can be so skewed that nodes in a subspace are already overwhelmed by the traffic designated to them, leaving them virtually no surplus power to share routing duties in enclosing spaces. Our scheduling algorithm has taken this into full account by pardoning heavily loaded sub-spaces (or the ones with meager power).

We modify an earlier e/CAN simulator by incorporating all the four algorithms described earlier. eCAN[13] is a prefixbased overlay capable of $\mathrm{O}(\ln N)$ routing performance, and this is achieved with simple extension to CAN[2]. In eCAN, the recursion in resolving routing is by zooming into topology sub-zones rather than shifting bits. However, our algorithm is immediately applicable to other prefix-based overlays such as Pastry [9], Tapestry [16] and Kademlia [5].

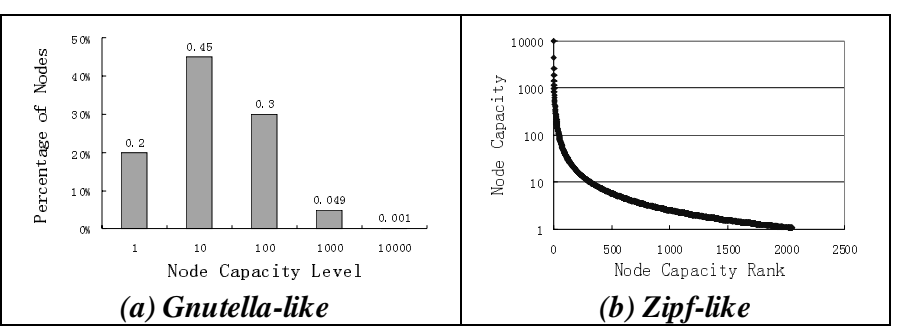

Figure 7: Capacity profile $(N=2 K)$

Two capacity profiles are used to model heterogeneity:

- Zipf-like: when sorted, the i-th node has capacity $10000 \cdot i^{-\beta}$, we choose $\beta$ be 1.2 by default.

- Gnutella-like: there are 5 levels of node, and the ith level has capacity $10^{\mathrm{i}-1}$, popularity in these levels are $20 \%, 45 \%, 30 \%, 4.9 \%$ and $0.1 \%$, going from level 1 to level 5 (see [10]).

The comparison of the two distributions for a $2 \mathrm{~K}$ node system is shown in Figure 7.

The eCAN configuration we use is equivalent to Pastry/Tapestry of $b=1$. We tested other configurations [15] and results are similar to those presented here. For each configuration (capacity profile, $N$ and other parameters), an experiment of 5 cycles is run. Each cycle starts with a complete reshuffling of the node capacities, then route $100 \mathrm{~N}$ times, during which load and capacity information are gathered. We then run the four algorithms to perform load balance. Finally another $100 \mathrm{~N}$ routings are performed and various statistics are collected again. This somewhat primitive setup allows us to gain sufficient insight of the algorithms; a more sophisticated one would include node join and leave events and mix SOMO traffics with normal routing, which we plan to conduct in the future.

We found that, in all configurations, load balance converges quickly in $O(\log N)$ time, and that after the full set of the algorithms are run, higher capacity nodes are taking more loads. Figure 8 and Figure 9 show a typical pair of results of the Gnutella-like and Zipf-like capacity distributions, respectively. Note the sharp difference before and after the load redistribution.

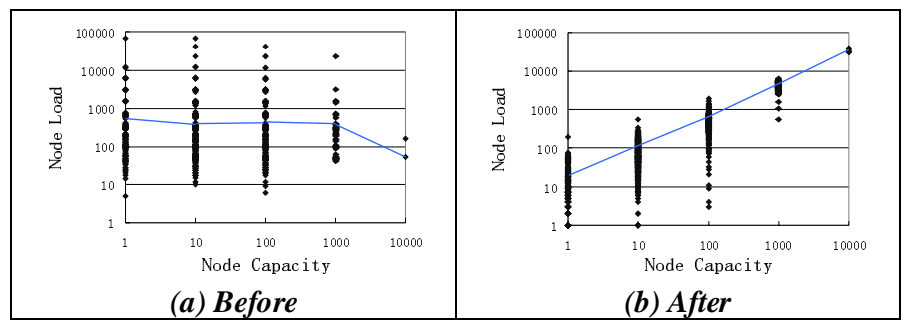

Figure 8: Results of $N=2 \mathrm{~K}$, Gnutella-like (the line corresponds to average load of a capacity level)

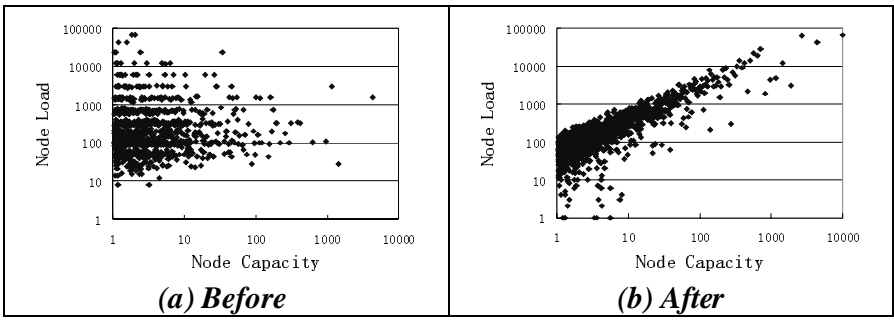

Figure 9: Results of $\mathrm{N}=2 \mathrm{~K}$, Zipf-like

\section{Related work}

Data overlay relies on the key property of the P2P DHT $([9][16][11][7][5][13])$ that an item with unique key can be reliably created and retrieved. To our knowledge, extending the principle of self-organizing to arbitrary data structure other than hash table and do it in a way that is agnostic to both semantics and performance of the hosting P2P DHT is new.

A pure "peer-to-peer" mindset will view hierarchy as forbidden word. We believe this is misleading as important functionalities such as aggregation and indexing [6][1] inherently imply a hierarchical structure. On this, SOMO bears the most similarity to Astrolabe [12], a peer-to-peer management and data mining system, for instance the use of hierarchies and aggregation. SOMO operates at the rudimentary data structure level while Astrolabe is on a virtual, hierarchical database. SOMO's extensibility is much like that of active network, whereas Astrolabe uses SQL queries. The marked difference is that SOMO is designed specifically on top of P2P DHT, for two reasons: 1) we believe P2P DHTs have established a foundation over which many other systems can be built and thus there is a need for a scalable resource management and monitoring infrastructure and 2) by leveraging P2P DHT (in fact, data overlay) the design and protocols of such infrastructure can be much simpler. Distributed, in-network query processing has also been investigated in apparently unrelated fields such as sensor network, though the emphasis there is quite different [2].

The other alternative to build an aggregation and dissemination tree would be to use the application-level multicasting tree such as the one proposed by Scribe [2] and Bayeux [18]. These trees are formed by joining routes from 
individual nodes to the root and, as a result, are unstructured as opposed to SOMO. The maintenance of the tree would either require each tree node to keep states in the form of pointers to its children, or let DHT nodes route to the root periodically to refresh the tree structure. In SOMO, every node is uniquely identified by the region that its report covers and therefore requires zero states. The structured nature of SOMO also allows queries to arbitrary sub-region of the total space, which would be otherwise impossible.

\section{Conclusion and Future work}

This paper makes several novel contributions: we describe how arbitrary data structures can be implemented on P2P DHT using the concept of data overlay; we designed and evaluated a self-organizing and robust metadata gathering and dissemination infrastructure SOMO. We have demonstrated how to balance routing traffic with node capacity in prefixbased overlay using both of these two techniques. Our future work includes more extensive study of these concepts.

\section{Acknowledgement}

The authors would like to thank Yu Chen and Qiao Lian for their insightful discussion. Dan Zhao helped to prepare this report as well. We also thank the anonymous reviewers for their useful comments.

\section{References}

[1] Adamic, L., Huberman, B., Lukose, R., and Puniyani, A. Search in Power Law Networks, Physical Review. E64(2001), 46135-46143

[2] Castro M., Druschel P., Kermarrec A., and Rowstron A. SCRIBE: A Large-scale and Decentralized Application-level Multicast Infrastructure. IEEE Journal on Selected Areas in Communications, Vol. 20. No 8. Oct. 2002

[3] Clarke, I., et al. Freenet: A distributed anonymous information storage and retrieval system. In Workshop on Design Issues in Anonymity and Unobservability. 2000. Berkeley, CA, USA.

[4] Madden, S and et al. TAG: A Tiny AGregation Service for AdHoc Sensor Networks. OSDI'02

[5] Maymounkov, P. and Mazieres D. Kademlia: a Peer-to-Peer Information System Based on the XOR Metric. In $1^{\text {st }}$ International Workshop on Peer-to-Peer Systems (IPTPS'02), (Cambridge, MA March 2002)

[6] Qin Lv and Sylvia Ratnasamy, Can Heterogeneity Make Gnutella Scalable? Proceedings of IPTPS 2002

[7] Ratnasamy, S., et al. A Scalable Content-Addressable Network. In ACM SIGCOMM. 2001. San Diego, CA, USA.

[8] Ratnasamy, S., et al. Location-Aware Overlay Construction and Server Selection. In Infocom. 2002.

[9] Rowstron, A. and P. Druschel. Pastry: Scalable, distributed object location and routing for largescale peer-to-peer systems. in IFIP/ACM Middleware. 2001. Heidelberg, Germany.

[10] Saroiu, S., Gummadi, K., and Gribble, S. A measurement study of peer-to-peer file sharing systems. In Proceedings of Multimedia Conferencing and Networking (San Jose, Jan. 2002)

[11] Stoica, I., et al. Chord: A scalable peer-to-peer lookup service for Internet applications. In ACM SIGCOMM. 2001. San Diego, CA, USA.

[12] Van Renesse, Robert and Birman Kenneth. Scalable Management and Data Mining using Astrolabe. Proceedings of IPTPS 2002.
[13] $\mathrm{Xu}$, Zhichen and Zhang, Zheng, Building Low-maintenance Expressways for $P 2 P$ Systems, available at http://www.hpl.hp.com/techreports/2002/HPL-2002-41.html, March 2002

[14] Zhang, Z., Turning P2P DHT into a Parallel Computing Utility. Submitted for publication.

[15] Zhang, Zheng, Shi, Shu-ming and Zhu, Jing. Self-balanced P2P expressway: when Marxism meets Confucian. MSR-TR-200272

[16] Zhao, B., Kubiatowicz, J.D., and Josep, A.D. Tapestry: An infrastructure for fault-tolerant wide-area location and routing. Tech. Rep. UCB/CSD-01-1141, UC Berkeley, EECS, 2001.

[17] Zhao, B., and et al. Brocade, Landmark Routing on Overlay Networks. In IPTPS'02

[18] Zhuang S.Q., Zhao B.Y., and Joseph A.D. Bayeux: An Architecture for Scalable and Fault-tolerant Wide-Area Data Dissemination, NOSSDAV'01, New York, USA 